\title{
Sonographic Findings of Polyneuropathy Associated With Cerebrotendinous Xanthomatosis: A Case Report
}

\author{
Jung Yoon Yoon, MD, Min-Wook Kim, MD, PhD, Hyun Jung Do, MD, \\ Dae-Hyun Jang, MD, PhD, Hee Won Lee, MD
}

Department of Rehabilitation Medicine, College of Medicine, The Catholic University of Korea, Seoul, Korea

\begin{abstract}
Cerebrotendinous xanthomatosis is a rare autosomal recessive disease that involves multiple organs, including the peripheral nervous system. The present study is the first to report the ultrasonographic findings of peripheral nerves in a patient with cerebrotendinous xanthomatosis. The patient presented with bilateral Achilles tendon enlargement and foot hypesthesia. Sonographic examination revealed hypoechoic, swollen peripheral nerves with enlarged bilateral Achilles tendons. Since the ultrasonographic findings revealed peripheral involvement, the diagnosis of cerebrotendinous xanthomatosis was established after laboratory and genetic studies along with clinical findings.
\end{abstract}

Keywords Ultrasonography, Polyneuropathies, Cerebrotendinous xanthomatosis

\section{INTRODUCTION}

Polyneuropathy is characterized by clinical sensory deficit, progressive weakness, and decreased tendon reflex in the extremities. There are several causes of polyneuropathy, including diabetes, toxins, hereditary or acquired demyelinating polyneuropathy, or cerebrotendinous xanthomatosis (CTX). CTX is a rare autosomal recessive disorder of lipid metabolism caused by mutation of the CYP27A1 gene [1]. This syndrome results in diverse manifestations, such as ataxia, dementia, epilepsy, and peripheral neuropathy, with multiple organ involvement including the central and peripheral nervous systems $[1,2]$.

Although previous studies have reported imaging findings of central nervous system involvement in CTX [1-4], ultrasonographic findings of peripheral nervous system involvement in CTX have not been described in the lit-

Received March 25, 2016; Accepted June 7, 2016

Corresponding author: Min-Wook Kim

Department of Rehabilitation Medicine, Incheon St. Mary's Hospital, College of Medicine, The Catholic University of Korea, 56 Dongsu-ro, Bupyeonggu, Incheon 21431, Korea. Tel: +82-32-280-5858, Fax: +82-32-280-5556, E-mail: minukkim@nate.com

ORCID: Jung Yoon Yoon (http://orcid.org/0000-0002-4703-9266); Min-Wook Kim (http://orcid.org/0000-0003-4505-809X); Hyun Jung Do (http:// orcid.org/0000-0001-7972-2548); Dae-Hyun Jang (http://orcid.org/0000-0001-8293-084X); Hee Won Lee (http://orcid.org/0000-0001-8367-7575).

(c) This is an open-access article distributed under the terms of the Creative Commons Attribution Non-Commercial License (http://creativecommons.org/ licenses/by-nc/4.0) which permits unrestricted noncommercial use, distribution, and reproduction in any medium, provided the original work is properly cited. Copyright (c) 2017 by Korean Academy of Rehabilitation Medicine 
erature. Here, we report ultrasonographic findings of polyneuropathy in a patient with CTX syndrome.

\section{CASE REPORT}

A 39-year-old man presented with a 19-year history of bilateral, slowly progressive, painful swelling of the Achilles tendon and a 1-year history of hypesthesia over the right sole without any trauma. The patient had no history of cataract or epilepsy, although he did have chronic diarrhea and had attended a special school due to learning disabilities.

Physical examination found a huge ovoid mass in bilateral Achilles tendons (Fig. 1A). The patient experienced no pain during rest or ankle motion. Muscle strength was normal, except for mild weakness in both ankles. A sensory test revealed that the patient had decreased sensation in both feet, especially to light touch. The deep tendon reflex was increased in both lower extremities. Babinski sign and ankle clonus were positive on both sides. Magnetic resonance imaging (MRI) of the ankle showed fusiform thickening of the Achilles tendon and an enlarged tibial nerve (Fig. 1B, 1C). The patient was mentally alert and he scored 25 points in the Mini-Mental State Examination with a very low IQ of 52 points.

Ultrasonography showed diffusely enlarged peripheral nerves in the lower limbs, upper limbs, and C5-C7 nerve roots. We also performed ultrasonographic evaluation of
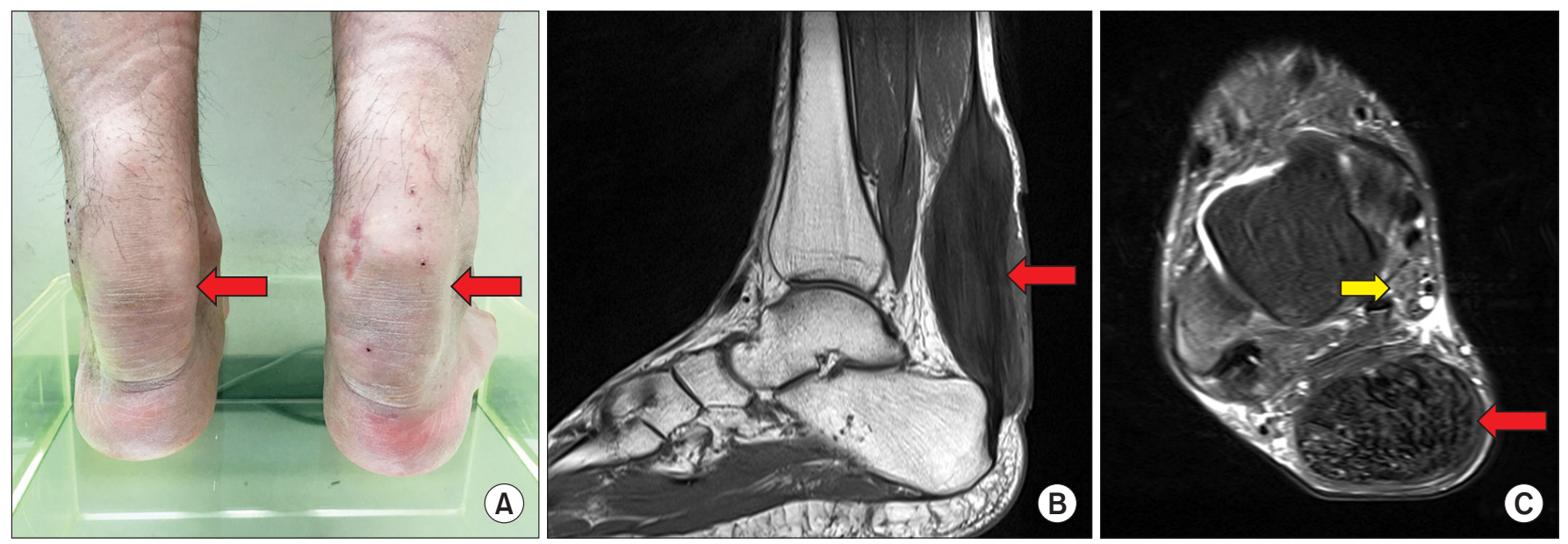

Fig. 1. Clinical photograph demonstrated bilateral fusiform enlargement of the Achilles tendon (red arrow) (A). A sagittal T1-weighted image (B) and an axial T2-weighted image of the ankle magnetic resonance imaging (C) showed a diffuse, hypointense mass occupying the entire Achilles tendon (red arrow) with a swollen tibial nerve (yellow arrow) behind the medial malleolus. The xanthoma measured $13.8 \mathrm{~cm}$ in length, $3.2 \mathrm{~cm}$ in anteroposterior diameter, and 4.4 $\mathrm{cm}$ in the transverse dimension.

Table 1. Cross-sectional area values of peripheral nerves in the patient with cerebrotendinous xanthomatosis and in the normal subject, measured by ultrasonography

\begin{tabular}{|llcc}
\hline \multirow{2}{*}{ Nerve } & \multicolumn{1}{c}{ Site } & \multicolumn{2}{c}{ Cross-sectional area $\mathbf{~ m m}^{\mathbf{2}}$ ) } \\
\cline { 3 - 4 } Tibial & Medial malleolus & Patient & Normal subject \\
\hline & 7 cm proximal to the medial malleolus & 25.7 & 6.8 \\
& Popliteal fossa & 19.4 & 4.4 \\
\hline \multirow{2}{*}{ Peroneal } & Fibular head & 48.0 & 19.8 \\
\hline Median & Carpal tunnel inlet & 18.9 & 9.9 \\
& Mid-forearm & 11.9 & 6.6 \\
& Antecubital fossa & 10.0 & 4.4 \\
\hline \multirow{2}{*}{ Ulnar } & Medial epicondyle & 16.7 & 5.1 \\
\hline
\end{tabular}


the peripheral nerves in a normal male subject of similar age and anthropometry. Cross-sectional areas (CSA) of the peripheral nerves were measured as shown in Table 1. Significantly larger CSA values were found in the patient with CTX than in the normal subject, for the posterior tibial nerve behind the medial malleolus and for the median nerve at both the wrist and the elbow, like the other peripheral nerves (Fig. 2).

Nerve conduction studies showed decreased sensory conduction velocity of the ulnar nerve on both sides (right, $43.7 \mathrm{~m} / \mathrm{s}$; left, $46.0 \mathrm{~m} / \mathrm{s}$ ) and decreased motor conduction velocity of the peroneal nerve (right, $37 \mathrm{~m} / \mathrm{s}$; left, $38.2 \mathrm{~m} / \mathrm{s}$ ). The posterior tibial nerve somatosensory evoked potential test (SEP) showed no response, while the median nerve SEP was normal. Brain MRI revealed mild cerebellar atrophy. T2-weighted image and fluid-attenuated inversion recovery (FLAIR) images of the brain showed areas of slightly high signal intensity in the bilateral fronto-parieto-occipital periventricular white matter (Fig. 3).

The results of laboratory tests, including serum total cholesterol, low density lipoprotein cholesterol, triglyceride, human leukocyte antigen B27, C-reactive protein, rheumatoid factor, antinuclear antibodies, and thyroid function, were normal, with the exception of cholestanol, which was high at $21.85 \mu \mathrm{g} / \mathrm{mL}$ (normal, $<3.71 \mu \mathrm{g} / \mathrm{mL}$ ).
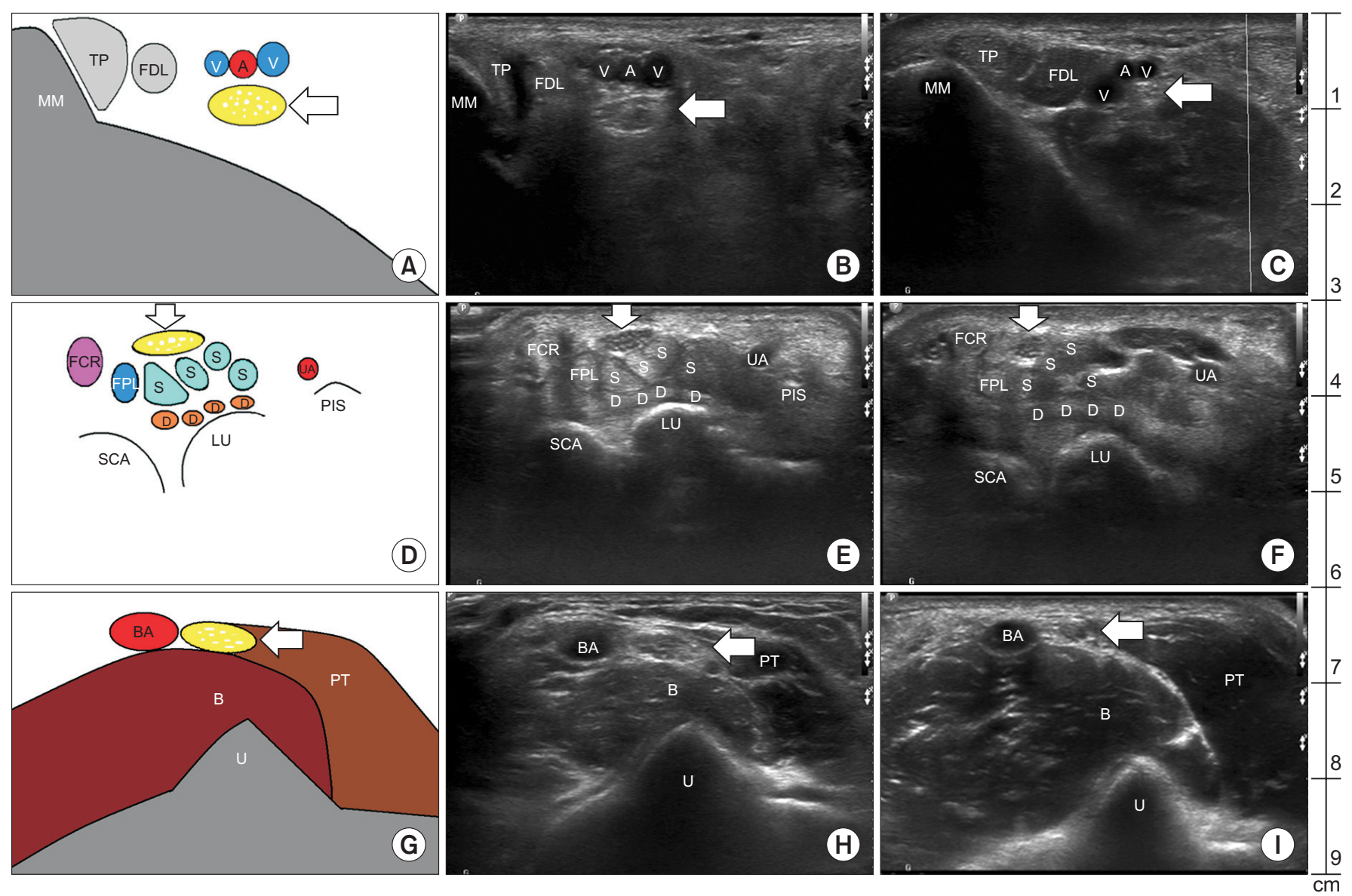

Fig. 2. Schematic illustrations show sonographic image of the patient (A, D, G). Transverse ultrasonography scan of the peripheral nerves in the patient $(\mathrm{B}, \mathrm{E}, \mathrm{H})$ and in a normal subject whose anthropometry was similar to that of the patient (C, F, I). In the patient, the tibial nerve (arrow) behind the medial malleolus was swollen, compared with that in the normal subject (A-C). The median nerve (arrow) at the wrist and elbow was also swollen, compared with that in the normal control (D-I). MM, medial malleolus; TP, tibialis posterior; FDL, flexor digitorum longus; A, posterior tibial artery; V, posterior tibial vein; SCA, scaphoid bone; PIS, pisiform bone; FCR, flexor carpi radialis; FPL, flexor pollicis longus; S, flexor digitorum superficialis; D, flexor digitorum profundus; UA, ulnar artery; BA, brachial artery; B, brachialis; PT, pronator teres; U, ulna bone. 

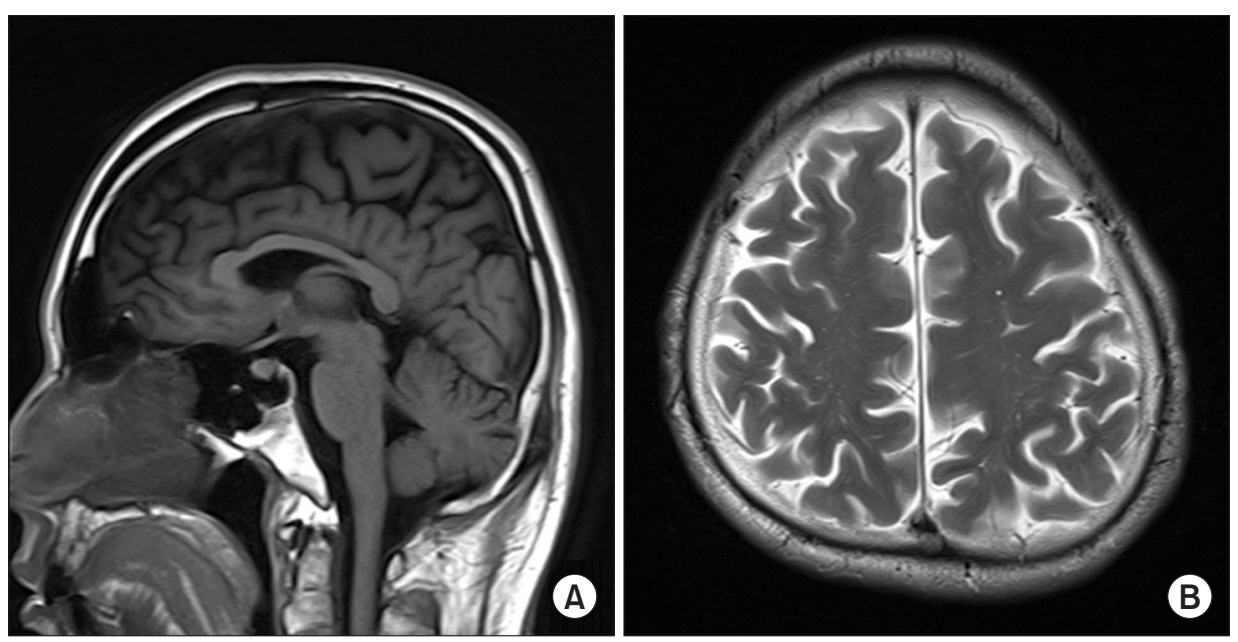

Fig. 3. On magnetic resonance imaging of the brain, the sagittal T1-weighted image showed mild shrinkage of the cerebellum (A) and the axial T2-weighted image showed slightly high signal intensity in the bilateral fronto-parietooccipital periventricular white matter (B).

Genetic analysis revealed two mutations in exon2 of the CYP27A1 gene: c.435G $>\mathrm{T}$ in the splice site and a missense mutation of c.379C $>$ T.

The above findings allowed us to make the diagnosis of CTX in the patient. The patient was treated with comprehensive physical therapy and $750 \mathrm{mg}$ /day of chenodeoxycholic acid.

\section{DISCUSSION}

A few previous reports have documented peripheral nervous system involvement in patients with CTX using a variety of measurement methods, including electrodiagnostic study, such as nerve conduction study and an electromyogram [3-6], sural nerve biopsy [3,6,7], and muscle biopsy [6].

However, our case report provides the first detailed ultrasonography evaluation in the literature showing diffuse peripheral nerve enlargement in a patient with CTX. On ultrasonographic examination, the CSA of the posterior tibial nerve was $150 \%$ to $220 \%$ greater than the normal reference value $[8,9]$. In addition, the CSAs of the other peripheral nerves were also significantly larger than the upper limits of the normal reference values [8$10]$.

CTX is a disorder that involves abnormality in cholesterol metabolism, which results in abnormal cholestanol accumulation in multiple organs, including nervous systems, and causing deterioration of their functions $[1,2]$. This defective bile acid synthesis may have caused neural damage. Based on the electrodiagnostic study, polyneuropathy in CTX could be categorized as the motor axonal, sensory-motor axonal, motor demyelinating, or sensorymotor demyelinating type [4-6]. Some authors reported that the predominant pathology of polyneuropathy in CTX is demyelination [4]; whereas the other authors reported that the axonal type was predominant $[5,6]$. In the literature, the primary pathological type of polyneuropathy in CTX is still a matter of debate. Altered lipid metabolism may cause a problem in myelin synthesis and the loss of myelin-axon integrity, leading to axonal degeneration [5]. Based on the nerve conduction study in our patient, the pathology was considered to be the sensory-motor demyelinating type. When aggravation of neural damage occurs, myelin-axon integrity is lost, and axonal damage may be observed in an electrodiagnostic study.

Nerve biopsy in patients with CTX reveals severe, diffuse loss of large myelinated fibers, clusters of small myelinated fibers, thin myelin sheaths, onion bulb formation (Schwann cell proliferation) [3,6], and lipid granules in Schwann cells [3]. In the biopsy of the proximal part of the sural nerve, huge onion bulbs have been found [6]. In our case, ultrasonography revealed diffuse enlargement of the peripheral nerves in the upper and lower limbs, from the distal to the proximal part. These findings are likely to be due to onion bulb formation and lipid deposits within Schwann cells.

In bilateral Achilles tendinopathy, primarily spondyloarthropathies such as ankylosing spondylitis should be ruled out. Bilateral Achilles tendon xanthoma might be observed in some hereditary diseases, such as familial hypercholesterolemia and sitosterolemia. Increased cholestanol level with normal cholesterol and sitosterol lev- 
els differentiates CTX xanthoma from familial hypercholesterolemia and sitosterolemia xanthomas. Neurologic symptoms are also an important factor in differentiating CTX from these two conditions, as they do not affect the central and peripheral nervous systems $[1,2]$.

Several electrodiagnostic studies have revealed polyneuropathy in $62.0 \%$ to $74.2 \%$ of patients with CTX [4-6]. This shows that polyneuropathy is a common feature of CTX. Therefore, checking for peripheral nerve abnormality is a crucial factor in the diagnosis of CTX. Most CTX patients show electrophysiological alteration at the subclinical stage [5]. Even when there are no symptoms or evidence of neuropathy in an electrophysiological study, nerve biopsy can reveal excessive de- and re-myelination [7]. In our case, nerve biopsy was not performed. However, on ultrasonographic examination, the peripheral nerves were found to be diffusely enlarged in both the upper and lower limbs, while the electrodiagnostic study showed minimal conduction slowing. In addition, the high level of cholestanol, the abnormality in brain imaging, and the genetic mutation led us to confirm the diagnosis of CTX.

This case report demonstrates that ultrasonography examination of peripheral nerve enlargement can aid in the diagnosis of CTX in patients with bilateral Achilles tendon xanthoma. Ultrasonographic evaluation can demonstrate peripheral nerve enlargement, even in the subclinical peripheral nerve involvement stage, in patients with CTX. Noninvasive and cost-effective ultrasonography nerve examination can be used as a screening test in bilateral Achilles tendon enlargement for making efficient differential diagnosis.

\section{CONFLICT OF INTEREST}

No potential conflict of interest relevant to this article was reported.

\section{REFERENCES}

1. Nie S, Chen G, Cao X, Zhang Y. Cerebrotendinous xanthomatosis: a comprehensive review of pathogenesis, clinical manifestations, diagnosis, and management. Orphanet J Rare Dis 2014;9:179.

2. Moghadasian MH. Cerebrotendinous xanthomatosis: clinical course, genotypes and metabolic backgrounds. Clin Invest Med 2004;27:42-50.

3. Wang Z, Yuan Y, Zhang W, Zhang Y, Feng L. Cerebrotendinous xanthomatosis with a compound heterozygote mutation and severe polyneuropathy. Neuropathology 2007;27:62-6.

4. Pilo B, de Blas G, Sobrido MJ, Navarro C, Grandas F, Barrero FJ, et al. Neurophysiological study in cerebrotendinous xanthomatosis. Muscle Nerve 2011;43:531-6.

5. Ginanneschi F, Mignarri A, Mondelli M, Gallus GN, Del Puppo M, Giorgi S, et al. Polyneuropathy in cerebrotendinous xanthomatosis and response to treatment with chenodeoxycholic acid. J Neurol 2013;260: 268-74.

6. Verrips A, van Engelen BG, ter Laak H, Gabreels-Festen A, Janssen A, Zwarts M, et al. Cerebrotendinous xanthomatosis: controversies about nerve and muscle: observations in ten patients. Neuromuscul Disord 2000;10:407-14.

7. Ohnishi A, Yamashita Y, Goto I, Kuroiwa Y, Murakami S, Ikeda M. De- and remyelination and onion bulb in cerebrotendinous xanthomatosis. Acta Neuropathol 1979;45:43-5.

8. Qrimli M, Ebadi H, Breiner A, Siddiqui H, Alabdali M, Abraham A, et al. Reference values for ultrasonograpy of peripheral nerves. Muscle Nerve 2016;53:538-44.

9. Scheidl E, Bohm J, Simo M, Bereznai B, Bereczki D, Aranyi Z. Different patterns of nerve enlargement in polyneuropathy subtypes as detected by ultrasonography. Ultrasound Med Biol 2014;40:1138-45.

10. Won SJ, Kim BJ, Park KS, Yoon JS, Choi H. Reference values for nerve ultrasonography in the upper extremity. Muscle Nerve 2013;47:864-71. 